\title{
Genderstern und Binnen-I
}

\section{$\mathrm{Zu}$ falscher Symbolpolitik in Zeiten eines zunehmenden Illiberalismus}

\section{Rudolf Stöber}

Eingegangen: 8. Oktober 2020 / Angenommen: 23. November 2020 / Online publiziert: 16. Dezember 2020

(C) Der/die Autor(en) 2020

Dieser Text soll und wird nicht jeder und jedem gefallen; er soll meinen Standpunkt verdeutlichen, zum Nachdenken und gegebenenfalls zum Überdenken der eigenen Haltung anregen. Zugleich ist er die Aufforderung zu einem fachgruppenübergreifenden Forschungsprojekt, das Rezeption und Wirkung von Genderstern (im folgenden: Gender-*) und Binnen-I untersucht.

In Kürze: Gender-* und Binnen-I sind derzeit sprachnormwidrig, die Debatte wurzelt in einem Missverständnis und produziert Kategorienfehler. Wer Gender-* und Binnen-I das Wort redet, muss sich fragen lassen: 1. Wie latent manipulativ, unausgewogen, latent ideologisch, polarisierend und zudem an falscher Stelle sprachsensibel möchte man sich ausdrücken? 2. Werden etwaige Nebenwirkungen angemessen bedacht? Wird nicht vielleicht aus gut gemeinten Gründen etwas schlecht Gemachtes, Dysfunktionales in die Welt gesetzt? 3. Möchte man mit allen, die Gender-* und Binnen-I benutzen, in einem Boot sitzen?

\section{Zu den Fakten}

Derzeit gelten Gender-* und Binnen-I als sprachnormwidrig. Es gibt eine zwischenstaatliche Einrichtung, den „Rat für deutsche Rechtschreibung“ (RdR). In ihm sitzen Vertreter Deutschlands, der Schweiz, Österreichs, Italiens, Belgiens und Luxemburgs. Der RdR gibt Empfehlungen zum amtlichen Regelwerk der Orthografie in den Mitgliedstaaten, die bei offiziellen Dokumenten von Ämtern und Behörden bedacht werden sollten. Der Rat beobachtet fortlaufend die Debatte: Gender-* und

Prof. Dr. R. Stöber $(\bowtie)$

Institut für Kommunikationswissenschaft, Otto-Friedrich-Universität Bamberg, An der

Weberei 5, 9647 Bamberg, Deutschland

E-Mail: rudolf.stoeber@uni-bamberg.de 
Binnen-I gelten dem Rat 2020 noch immer als Abweichung von der Norm. Gegen gendergerechte Sprache hat der RdR nichts einzuwenden.

Der RdR forderte 2018 dazu auf, sechs Kriterien zu beachten: die Kriterien ,der sachlichen Korrektheit, der Verständlichkeit und Lesbarkeit, der Vorlesbarkeit, der Rechtssicherheit und Eindeutigkeit und der Übertragbarkeit“ auf die verschiedenen Länder. Der RdR hielt fest: „Die Verwendung des Binnen-I ist von den amtlichen Regeln der Groß- und Kleinschreibung [...] nicht abgedeckt [...]. Sowohl die X-Form als auch beide Formen des Gender-Gap (statischer wie dynamischer Unterstrich) als Kennzeichnung der Aufhebung binärer Geschlechtsvorstellungen sind lediglich in bestimmten Gruppen und Communities verbreitet und entsprechen zum allergrößten Teil nicht den Kriterien, die nach Auffassung des Rats an korrekte Texte gestellt werden müssen [...]. Dies gilt in deutlich geringerem Maße für den Asterisk, das Gender-Sternchen, das zunächst in Hochschulen und ihrem Umfeld entwickelt und als Kurzform einer Männer wie Frauen umfassenden Schreibung genutzt wurde“ (RdR AG „Geschlechtergerechte Schreibung“ 2018, S. 9f.).

Die Universitäten sollten diesen Empfehlungen folgen; stattdessen verstoßen ihre Vertreter zunehmend dagegen. Privatpersonen sind nicht verpflichtet, sich daran zu halten; Angehörige von Universitäten hingegen schon - zumindest, wenn sie als solche auftreten, z. B. indem sie die Uni-Mail-Adresse als Kontaktadresse in Aufsätzen angeben. Stattdessen verfassen universitäre Genderbeauftragte Handreichungen zu gendergerechter Sprache, die bisweilen kuriosen Sprachverkomplizierungen das Wort reden.

Alle sollten zumindest den Duden beachten: Gendergerechte Sprache ist Dudenkonform. Aber es gibt keine Lemmata mit Gender-* und Binnen-I. Zwar nimmt der Duden zu Gender-* und Binnen-I eine weiche Position ein und ist überdies nicht sprachnormsetzend, sollte aber allgemein als Referenz akzeptiert werden.

Die Frage ist, warum in den letzten Jahren um Gender-* und Binnen-I eine Debatte losgetreten wurde. Offensichtlich aus zwei unterschiedlichen Gründen: a) aus einem Missverständnis und b) aus wahrnehmungspsychologischen Überlegungen, die in dem Missverständnis wurzeln.

Offenkundig stand am Anfang ein Missverständnis: Die gegenwärtige Verwirrung ist Folge einer weit zurückliegenden Übersetzung. In der Grammatik ist lateinisch von genus die Rede, wenn die Wortgattung bezeichnet werden soll. Übersetzt wurde das lateinische Wort genus ab dem späten Mittelalter (!) mit „Geschlecht“; gemeint war Gattung. Geschlecht hatte bis dahin zwei Bedeutungen: Es bezeichnete eine Abstammungs- oder Ahnenfolge, insbesondere beim Adel sprach man von Geschlechtern. Geschlecht bezeichnet zudem die Unterscheidung von Mann und Frau, lateinisch sexus. Warum hätte das Lateinische zwei Wörter gebraucht, wenn sie das Gleiche bezeichnen? Das Lateinische unterscheidet die beiden Wörter, weil sie Unterschiedliches benennen: genus ist nicht gleich sexus. Das ist in der Linguistik allgemein anerkannt.

Zunächst hatte die Übertragung von genus keine Konsequenzen. Erst in unserer Gegenwart wird die Sprache durch die Identifikation von sprachlicher Gattung mit biologischem Geschlecht aufgeladen - weil man es nicht mehr besser weiß oder wissen will. Die Genus-Zuweisung ist willkürlich: der Stuhl, die Stuhllehne, das Stuhlbein - das Genus hat nichts zu bedeuten, wenn es um Unbelebtes geht. Nur 
Lebewesen haben ein natürliches Geschlecht. Die unbedingte Gleichsetzung ge$n u s=$ sexus ist ein Kategorienfehler; hier wird Inkommensurables über einen Kamm geschoren. Das ist bestenfalls Zeichen von Halbbildung und spottet jeder Differenzierung, die der Gender-* doch eigentlich markieren soll.

Allerdings, und dabei sind wir bei der wahrnehmungspsychologischen Argumentation, gibt es eine Reihe von Untersuchungen, die belegen (wollen), dass die generische Pluralbildung insbesondere bei Frauen den Eindruck erweckt, Frauen seien im gesellschaftlichen Leben unterrepräsentiert. Eine Studie, die dieser Auffassung zuneigt, fasst zusammen: „Viele Experimentalstudien zeigen, dass die Ambiguität maskulin-geschlechtsübergreifender Referenzen in vielen Kontexten weibliche Personen in der mentalen Repräsentation zurücktreten lässt“" (Kotthoff 2020, S. 6). Ähnlich argumentierten schon Braun et al. (2005). Blake und Klimmt kamen zu der Einschätzung, dass das generische Maskulinum zur Unterschätzung, das Binnen-I zur Überschätzung des Frauenanteils in untersuchten Feldern führe; nur die gemischte Geschlechterbezeichnung ,führte zu einer angemessenen Einschätzung des Anteils am Ereignis beteiligter Frauen“" (Blake und Klimmt 2010, S. 298). ${ }^{1}$

\section{Meine Position}

Als politisches Motiv hinter der Sprachnormverletzung steht das Bemühen, die Emanzipation voranzutreiben - das konnte ich wiederholt in Diskussionen bemerken. Doch wo endet die aufrichtig gemeinte Bemühung um mehr Emanzipation? Wo beginnt die Gedankenpolizei?

Kritisch wurde es für mich in diesem Frühjahr, als unaufgefordert in einen meiner Texte Gender-* und Binnen-I eingefügt wurden. Bezeichnenderweise geschah das in einem Text für die 2. Auflage eines Handbuchs, das im Springer-Verlag erscheinen wird. Erst auf meine Drohung hin, den Text zurückzuziehen, wurde der Ursprungszustand wiederhergestellt.

Spätestens seither sehe ich die manipulative Tendenz der symbolischen Auseinandersetzung. Der heraufdämmernde, wachsende Illiberalismus, den ich fürchte, legitimiert sich aus der Beobachtung eines doppelten Zusammenhangs: des Zusammenhangs von Sprache und Denken (1) und des Zusammenhangs von Denken und Wirklichkeit (2). An beidem ist etwas dran.

Ad 1) Fraglos hängen Sprache und Denken zusammen. Schon Heinrich von Kleist wusste um die ,,allmähliche Verfertigung der Gedanken beim Reden“ (Kleist 1925). George Herbert Mead sah die Evolution sprachlicher, kognitiver und sozialer Fähigkeiten als Co-Evolution. Den Ausdruck kannte er noch nicht, die moderne Evolutionstheorie bestätigt seine damaligen Hypothesen gleichwohl (vgl. Mead 1968). Die These von Sapir und Whorf, dass nur gedacht werden könne, was auszusprechen ist, hat sich trotz mancher Einschränkung cum grano salis bestätigt (vgl. Sapir 1961;

\footnotetext{
1 Die Untersuchung kam aber implizit zu dem Ergebnis, dass selbst bei der Verwendung des generischen Maskulinums der Anteil der Feuerwehrfrauen um mindestens das Doppelte, bei gegenderten Formulierungen sogar dreifach überschätzt wurde (vgl. Blake und Klimmt 2010, S. 301).
} 
Whorf 1963; Crystal 1993, S. 15). So weit, so gut: Der Zusammenhang von Sprache, Denken und Sozialität scheint für Gender-* und Binnen-I zu sprechen.

Es verhält sich leider genau umgekehrt: Sprache beeinflusst unser Denken als gewachsenes Proto-Medium - unreflektiert, nicht intentional. Als soziale Wesen benutzen wir Sprache als wichtigstes Verständigungsmittel. Sprache ist die bedeutendste mediale Institution. Wir benutzen sie ständig, wir denken nicht über jedes Wort nach, das wir sagen. Das macht das subkutane Beeinflussungspotenzial aus, das dem Sprachgebrauch unterliegt.

Wer hingegen bewusst sprachpolitisch eingreift, um via Sprache Denken zu lenken, manipuliert. Bei Victor Klemperer (1996), Dolf Sternberger et al. (1957) oder George Orwell (1949) lässt sich viel über die Gefährlichkeit der Gedankenmanipulation via Sprachlenkung lesen. Die Diskussion um Wording und Framing anlässlich des ARD-Gutachten von Elisabeth Wehling (2017) zeigt, dass wir längst auf der schiefen Ebene der Manipulation stehen und wo die Sprache zu rutschen beginnt. Wer die Institution Sprache beschädigt, darf sich über weitergehende Angriffe auf die Institution der Massenmedien nicht beklagen.

Nun wird niemand all jenen, die Gender-* und Binnen-I benutzen, Nähe zu Faschismus, Nationalsozialismus oder Kommunismus vorwerfen. Manche Nutzer aber zeigen genau diese Nähe. Ich möchte den Kolleginnen und Kollegen, die in unseren Zeitschriften publizieren, diese Nähe nicht unterstellen. Ich will nicht einmal den meisten Nutzern von Gender-* und Binnen-I außerhalb unseres Fachs unterstellen, bewusst manipulieren zu wollen. Aber: Soweit anzunehmen ist, dass die Lenkungswirkung zweiter Ordnung nicht angemessen reflektiert wird, soweit nur dem Trend eines bestimmten Milieus gefolgt wird, soweit man sich nur den Anschein der Fortschrittlichkeit geben will, soweit ist der Gebrauch von Gender-* und Binnen-I bestenfalls unbedacht, selbstgleichschaltend und latent manipulativ. Und das lehne ich mit Nachdruck ab.

Auch denjenigen, die sich offen und bewusst dazu bekennen, mittels Sprache das Denken beeinflussen wollen, werfe ich keinesfalls Nähe zu irgendeinem politischen Extremismus vor. Aber sie sollten sich zumindest selbst die Frage stellen, ob der Preis, den sie werden zahlen müssen, bzw. der Preis, den sie der Gesellschaft auferlegen, nicht vielleicht zu hoch ist. Ich kann mir nicht helfen: Manipulation, selbst solche aus ehrenwerten Motiven, bleibt Manipulation. Wollen sich die Sprachenund Gedankenmanipulateure vorwerfen lassen, die Gesellschaft mit billiger Symbolpolitik zu indoktrinieren und zu polarisieren? Gerade die deutsche Kommunikationswissenschaft sollte aufpassen: Vorgänger des Fachs wie Hans Amandus Münster oder die Äbte im Leipziger Roten Kloster hatten mit manipulativen Volksverführern paktiert und sich dem jeweiligen Zeitgeist angedient. Den bewusst manipulativen Einsatz von Sprache lehne ich mit noch größerem Nachdruck ab.

Um einem Totschlagargument gleich vorzubeugen - ich setze NS- und DDRDiktatur keineswegs gleich. In einem Punkt stimmt die Gleichsetzung aber auf jeden Fall: Beide waren, wie alle ideologiegetriebenen Diktaturen, doktrinär, manipulativ und wollten die Menschen zu ihrem Glück zwingen. Das endet in der Regel mit der Aufrichtung von Guillotinen.

Man mag einwenden, dass sich Wissenschaftler zu (sprach-)politischen Fragen nicht äußern sollten. Doch wer so argumentiert, macht es meines Erachtens nur 
schlimmer: Denn damit wird Experten die Expertise in dem Moment abgesprochen, in dem sie nicht mehr zur eigenen Anschauung passt. Wer die linguistische Expertise als irrelevant abtut, betreibt das Geschäft jener Populisten, welche die Experten und Wissenschaft generell diskreditieren. Damit wird indirekt an dem Ast gesägt, auf dem die Wissenschaft sitzt. Als scientists sollten wir aufpassen. Ich habe bewusst das englische Wort verwendet, weil der Streit v. a. in deutscher Sprache aufgeladen ist und energisch ausgetragen wird. Wer das sprachwissenschaftliche Argument in Abrede stellt, bekundet schon damit politische Absichten. Und gegen andere Formen der ,gendergerechten Sprache“ wende ich mich keineswegs.

Ad 2) Die Absicht, über die Sprache das Denken zu beeinflussen, speist sich vermutlich aus der beobachteten Wechselwirkung zwischen Denken und Realität. Beide hängen unbestreitbar zusammen. Allerdings nicht kausal und unidirektional, sondern wie ein Moebius-Band: Ununterscheidbar verkehren sich Unter- und Oberseite. Unter- und Überbau, mit Marx gesprochen, lassen sich zwar analytisch trennen, in der sozialen Wirklichkeit hingegen nicht. Was wir denken, wird automatisch Teil unserer Realität; die wahrgenommene Realität wirkt simultan auf unser Denken.

Nun mag, wer es schlicht sieht, glauben, mit Symbolen die Realitäten ändern und mit Gender-* und Binnen-I für Emanzipation sorgen zu können. Man mag sich daher für fortschrittlich halten. Ich halte es eher für magisches Denken, das auf einem Irrtum beruht: Realitäten, hier reale Diskriminierungen, sind durch „Neusprech“ (George Orwell) nicht aus der Welt zu schaffen. Wer glaubt, mit Gender-* und Binnen-I die Realitäten zu ändern, weil beides das Denken ändere, wer bewusst Sprache manipuliert, um zunächst das Denken zu ändern und damit die Emanzipation voranzutreiben, wacht vielleicht eines Tages erstaunt auf, weil etwas ganz anderes eingetreten ist: An die Stelle wünschenswerter Emanzipation sind Gender-* und Binnen-I getreten - und dabei ist es geblieben.

Oder aber, Gender-* und Binnen-I werden als Symbole gesellschaftlicher Polarisierung wahrgenommen. Für mich steht fest, mit dem Kampf um die Zeichen wird Symbolpolitik betrieben, Politik wird auf einen Nebenkriegsschauplatz verlagert. Wir werden sehen, was außer Sprachverhunzung und Polarisierung der Gesellschaft dabei „rumkommt“. Und sollten diejenigen, die Gender-* und Binnen-I verwenden, in Abrede stellen, über Sprache das Denken und darüber die Realität beeinflussen zu wollen, müssen sie sich fragen lassen, warum sie dann die Institutionen Schrift und Sprache beschädigen?

Ich gestehe gerne zu, dass mein Plädoyer gegen Gender-* und Binnen-I auch eine Geschmacksfrage ist. Darüber lässt sich bekanntlich streiten. Die Befürworter (generischer Plural) müssen sich aber die Frage stellen, warum sie in die schöne deutsche Sprache ein Sonderzeichen aus der technischen Welt der Computer-Sprache einführen wollen, um eine Trivialität anzuzeigen: die Trivialität, dass Menschen verschieden sind. Es scheint mir in dem Zusammenhang bemerkenswert, dass Diversität mit einem einheitlichen Zeichen abgebildet werden soll. Zugespitzt: Man erkauft den Hinweis auf eine Selbstverständlichkeit mit schriftsprachlicher Verarmung.

Warum spreche ich nicht nur von (latent) manipulativ, sondern auch von (latent) ideologisch? Unterstellen sich nicht die Befürworter und Gegner von Gender-* und Binnen-I wechselseitig, die jeweils anderen seien die Ideologen? Behaupten nicht 
beide Seiten, eine vernünftige Weltanschauung zu vertreten? Auf den ersten Blick scheinen beide Positionen in gleicher Weise unter Ideologieverdacht zu stehen scheinen beide Schreibvarianten in ähnlicher Weise ideologisiert zu sein.

Wer Gender-* und Binnen-I bewusst einsetzt, unterstellt den Gegnern, ideologisch ,patriarchalische“ Verhältnisse konservieren zu wollen. An dem Vorwurf ist sicherlich eines richtig: Wer wie ich für die historisch gewachsene Sprache plädiert, ist in dieser Hinsicht konservativer. Doch wer Regeln befolgt, muss sich nicht rechtfertigen, steht aber inzwischen unter latentem Rechtfertigungsdruck. Das ist bezeichnend und bestätigt in jedem einzelnen Fall von Rechtfertigungszwang das inzwischen in bestimmten Milieus verbreitete illiberale Klima. Oder anders: Wer sich an Regeln hält, muss das nicht begründen. Wer hingegen Sprache bewusst verändern will, sollte sich rechtfertigen (müssen). Darum nochmals: Symbolpolitik kostet nichts, sie ist wohlfeil und billig. ,,Teure Signale“ liefert hingegen nur das Verhalten. Man messe die Anhänger tradierter Schreibweise besser an ihren Aktionen als an der Verwendung des generischen Plurals.

Und wie steht es um die Ideologie von Gender-* und Binnen-I? Schaut man sich die Verwendungspraxis des Gender-* und Binnen-I an, wird e silentio deutlich, dass sie unausgewogen gebraucht werden. Gender-* und Binnen-I werden fast ausschließlich im positiven Zusammenhang verwendet. Zumeist kommen bei negativ konnotierten Wörtern weibliche oder gegenderte Formen nicht vor. NB.: Bert Brecht hat einmal eine weibliche Form verwendet, obwohl er eine männliche meinte: „Daß die Völker nicht erbleichen/ Wie vor einer Räuberin/ Sondern ihre Hände reichen/ Uns wie andern Völkern hin“ (Brecht 2005 [1950], S. 396f. und 507f.). Das stammt aus der 2. Strophe seiner „Kinderhymne“, seines Gegenentwurfs zur Becher-Hymne, der späteren Nationalhymne der DDR. Nun war Bert Brecht bekannterweise links und ein Macho. Wer ihm aber Misogynie unterstellen wollte, liegt falsch, denn offenkundig hatte er zur „Räuberin“ aus Gründen des Reims gegriffen. Wer wollte ihm das ankreiden? Wenn man Brecht, was ihn posthum wohl kaum stören würde, keinen Vorwurf machen will, sollte man auch jenen, die den generischen Plural verwenden, Frauenfeindlichkeit nicht unterstellen.

Zurück zur Verwendungspraxis von Gender-* und Binnen-I: Es gibt keine VerbrecherInnen oder Verbrecher*, Vergewaltiger* oder VergewaltigerInnen, Mörder* oder MörderInnen. Tucholskys ,SoldatInnen sind Mörder*“? Nicht vorstellbar! Wer wollte aus Schillers Räuber die Räuber*_Innen machen? Man sollte sich fragen, ob die selektive Verwendung nicht zumindest latent manipulativ-ideologisch zu nennen ist? Wollten die Anhänger*_Innen der gendergerechten Schreibe diesen Ideologieverdacht ausräumen, sollten sie konsequent bleiben. Doch wie sähen dann die Texte aus? Und was wäre gewonnen? Welch unglaubliche sprachliche Verarmung würde betrieben?

Was also soll mit Gender-* und Binnen-I ausgedrückt werden? Individualität und Diversität. Beides ist trivial, beides ständig zu markieren beleidigt den gesunden Menschenverstand und die vorhandene Toleranz. Wenn meine Intelligenz und meine Toleranz permanent unterschätzt werden, weil man glaubt, mich ständig an Selbstverständlichkeiten erinnern zu müssen, ärgert mich das irgendwann. Da es außerdem unmöglich ist, jeder möglichen Empfindlichkeit im Voraus zu entsprechen, weiß niemand ganz genau, wie in Zukunft die gegenderten Einheitstexte gelesen 
werden. Vielleicht als Lachnummer? Vielleicht als datumsloser Zeit(geist-)stempel? Sollen die deutschen Klassiker, sollen auch die Texte des klassischen Journalismus, sollen die Texte einer Anita Augspurg, Margret Boveri, Gabriele Tergit, eines Heinrich Heine, Kurt Tucholsky, Theodor Wolff oder Rudolf Augstein den Empfindlichkeiten und der PC-Kontrolle unterworfen werden? Die Nutzer*_Innen der aufdringlichen Markierung sollten ihren Sprachgebrauch auf aktuelle wie zukünftige Wirkungen einmal überdenken. Es ist schon jetzt auffällig, dass Rechtsradikale ihre anonymen Hassmails an „Scheiß-Aktivist*_Innen“ richten. Die Stigmatisierung wird schon umgedreht! Das passt zu der linguistischen These, dass Wörter mit extrem positiver oder negativer Konnotation nicht selten radikalen Umwertungen unterliegen. Man betrachte nur einmal den mehrfachen Bedeutungswechsel des Wortes ,geil“ im Laufe der Neuzeit.

Mich treibt weit mehr als ästhetisches Unbehagen: Erstmals seit den ironischen Anführungszeichen der Nazis, die Victor Klemperer nachdrücklich beklagte und die Goebbels, Hitler, Rosenberg und Konsorten so häufig einsetzten, wird in die Form der Sprache selbst eingegriffen, um (durchaus ehrenwerte) Ziele zu verfolgen. Die manierierte Gleichmacherei von Gender-* und Binnen-I ist mehr als eine semantische Neuschöpfung. Dass sie aus Unkenntnis entstanden ist, sei geschenkt. Ob sie das vermutlich angestrebte Emanzipationsziel verfehlt, wird die Zukunft erweisen. Die Auswege, die die gendergerechte Sprache da bisweilen findet, sind Kuriosa der Entdifferenzierung. Es ist nicht das Gleiche, von Studenten und von Studierenden zu sprechen. Student ist man auch in den „Semesterferien“, Studieren aber ist Tätigkeit; Studierende können nicht Party feiern, Studenten sehr wohl.

Hinzu kommt jedoch etwas, das mich mindestens so stark wie das Gendern umtreibt: der allgemeine Sprach-, Bildungs- und Kenntnisverfall. Es ist problematisch, wenn immer mehr Studienanfänger die deutsche Sprache in Orthografie und Interpunktion nicht mehr richtig beherrschen, wenn die Zeitenfolge ihnen ein Rätsel ist, wenn das einzig genutzte Tempus das dramatische Präsens ist, wenn Präpositionen falsch verwendet werden, schwache Verben die Texte regieren, sich der Modus in die terra incognita verirrt. NB.: Leider findet die Sprachverarmung schon Eingang in die Nachrichtensendungen der öffentlich-rechtlichen Sender, insbesondere von ZDF und 3Sat. Auch kommunikationswissenschaftliche Publikationen könnten sich etwas mehr Mühe geben, denn sie dienen den Studenten als Referenzen und Vorbilder.

Universität wie Wissenschaft im allgemeinen und die Kommunikationswissenschaft im besonderen werden sich kaum dadurch profilieren, dass sie schlechtem, falschem und verarmendem Deutsch Vorschub leisten, dass sie die Sprache durch affektierte Kunstpausen und die Schrift durch unästhetische Zeichen verunstalten. Zugleich wird heutzutage von Qualitätsmanagement, Human Resources, HiWis, Kollaboration und anderem gesprochen, ohne dass an die menschenverachtenden Konnotationen oder gar an die totalitären Bezüge dieser Wörter gedacht wird. Diesem unsensiblen Sprachgebrauch sollte man sich einmal zuwenden. 


\section{Zuletzt ein Vorschlag}

Damit bin ich beim letzten Punkt: Es sollte die kommunikationswissenschaftlichkritische Frage erlaubt sein, ob Gender-* und Binnen-I bei den Rezipienten so ankommen, wie sie wirken sollen? Denn der „Text konstituiert sich im Leser“ (Iser 1976). Es ist durchaus denkbar, dass Rezipientinnen und Rezipienten außerhalb intellektueller und wissenschaftlicher Milieus eher ablehnend reagieren und Texte und Sprechakte mit den genannten Manierismen als elitäre Sondersprache wahrnehmen. Es ist nicht auszuschließen, dass die Marotte zur Spaltung der Gesellschaft weiter beiträgt. Es wäre ein nützlicher Beitrag der Wirkungs- und Rezeptionsforschung zu prüfen, ob dem so ist: Wie kommen Gender-* und Binnen-I bei Manuela Musterfrau und Markus Mustermann an? Das wäre mit einem einfachen experimentellen Design zu testen. Man müsste Texte mit und ohne die Markierung einer Experimentalund einer Kontrollgruppe vorlegen und danach fragen, welche (ansonsten gleichlautenden) Texte als sachkundiger, glaubwürdiger etc. wahrgenommen werden? Bei welchen man mehr, bei welchen geringere Eigeninteressen der Verfasser vermutet? Welcher Text hat besser, welcher weniger gut gefallen? Man sollte empirisch prüfen, wer auf generische Pluralbildung und wer auf Gender-Stern und Binnen-I empfindlich reagiert? Wie viele, in welcher Altersstufe, mit welchem Bildungshorizont und von welchem Sozialstatus sind Kritiker der einen und der anderen Variante? Welche Texte werden besser erinnert? Werden eher die sprachlichen Manierismen oder die inhaltlichen Sachaussagen behalten? Welche Form der Schriftsprache bildet die Realität besser $\mathrm{ab} ?^{2}$

$\mathrm{Zu}$ all den Fragen gibt es schon vereinzelte Studien; aber sie widersprechen sich. Eine ältere, methodisch kritisierte Studie kommt zu der Einschätzung, dass ,die Ergebnisse jedoch insgesamt [belegen], dass geschlechtergerechte Texte ähnlich erfolgreich verarbeitet werden können wie Texte mit generisch maskulinen Bezeichnungen“. Die tabellarisch gelisteten Einzelergebnisse weisen hingegen auf, dass die generischen Textvarianten insgesamt die besseren Ergebnisse zeigen (vgl. Braun et al. 2007, S. 188). Eine Replikationsstudie mit sehr geringer Fallzahl kommt zu dem entgegengesetzten Urteil: „Contrary to [Braun et al. 2007] we found a nearly significant difference between text versions in case of information recall [...]. Participants recalled less information in the gender-fair condition." (Beller und Kazazi 2013, S. 6)

Um ideologischer Einfalt zu begegnen, sollten sich Vertreter (Vorsicht: generischer Plural) verschiedener Fachgruppen daran beteiligen: Medien, Öffentlichkeit und Geschlecht, Mediensprache - Mediendiskurse, Rezeptions- und Wirkungsforschung, Kommunikations- und Medienethik, Kommunikationsgeschichte ... Am besten die DGPuK in ganzer Breite. Auch die Medienökonomie, denn es dürfte vielleicht auch interessieren, wie viel an Ressourcen mehr oder weniger verbraucht wird, wie viele Zeilen geschunden und Sendezeit verschwendet wird, wenn ständig

\footnotetext{
2 Gerade weil ich den Autoren im Grundsatz zustimme, überzeugt mich die Argumentation von Blake und Klimmt zumindest da nicht, wo gendergerechte Formulierungen offensichtlich zu krasser Fehleinschätzung der tatsächlichen Verhältnisse führen, z. B. bei den Feuerwehrfrauen: Nachrichtensprache sollte nicht die Realität verzerren (vgl. Blake und Klimmt 2010, S. 301).
} 
in allen Varianten gegendert wird. Was wird an Informationsgewinn geliefert? Was geht an Informationen verloren?

Meine Mutmaßung ist, dass Gendern die Mehrzahl der Bevölkerung kaum interessiert; dass das Gendern in Schrift und Sprache außerhalb bestimmter Milieus eher auf Ablehnung stoßen wird; dass zum infamen Vorwurf der „Lügenpresse“ irgendwann noch der satirische Vorwurf des „Stolper-Rundfunks“ kommen wird. Meine Mutmaßung ist, dass durch die penetrante Redundanz von Trivialitäten neben der Sprach- auch eine Informationsverarmung eintreten wird. Und mit Rechtsradikalen, die gegen „Scheiß-Aktivist*_Innen“ pöbeln, möchte ich nicht einmal zeichentheoretisch in einem Boot sitzen.

Funding Open Access funding enabled and organized by Projekt DEAL.

Open Access Dieser Artikel wird unter der Creative Commons Namensnennung 4.0 International Lizenz veröffentlicht, welche die Nutzung, Vervielfältigung, Bearbeitung, Verbreitung und Wiedergabe in jeglichem Medium und Format erlaubt, sofern Sie den/die ursprünglichen Autor(en) und die Quelle ordnungsgemäß nennen, einen Link zur Creative Commons Lizenz beifügen und angeben, ob Änderungen vorgenommen wurden.

Die in diesem Artikel enthaltenen Bilder und sonstiges Drittmaterial unterliegen ebenfalls der genannten Creative Commons Lizenz, sofern sich aus der Abbildungslegende nichts anderes ergibt. Sofern das betreffende Material nicht unter der genannten Creative Commons Lizenz steht und die betreffende Handlung nicht nach gesetzlichen Vorschriften erlaubt ist, ist für die oben aufgeführten Weiterverwendungen des Materials die Einwilligung des jeweiligen Rechteinhabers einzuholen.

Weitere Details zur Lizenz entnehmen Sie bitte der Lizenzinformation auf http://creativecommons.org/ licenses/by/4.0/deed.de.

\section{Literatur}

Beller, J., \& Kazazi, J. (2013). Is there an effect of gender-fair formulations in the German language? Journal of Unsolved Questions, 3(1), 5-8.

Blake, C., \& Klimmt, C. (2010). Geschlechtergerechte Formulierungen in Nachrichtentexten. Publizistik, 55, 289-304.

Braun, F., Sczesny, S., \& Stahlberg, D. (2005). Cognitive Effects of Masculine Generics in German: An Overview of Empirical Findings. Communications, 30, 1-21.

Braun, F., Oelkers, S., Rogalski, K., et al. (2007). „Aus Gründen der Verständlichkeit ...“: Der Einfluss generisch maskuliner und alternativer Personenbezeichnungen auf die kognitive Verarbeitung von Texten. Psychologische Rundschau, 58(3), 183-189.

Brecht, B. (2005). Ausgewählte Werke in sechs Bänden. Bd. 3. Berlin: Suhrkamp.

Crystal, D. (1993). Die Cambridge Enzyklopädie der Sprache. Frankfurt a. M.: Campus.

Iser, W. (1976). Der Akt des Lesens. Theorie ästhetischer Wirkung. München: Fink.

Kleist, H. v. (1925). Über die allmähliche Verfertigung der Gedanken beim Reden. In H. v. Kleist (Hrsg.), Sämtliche Werke. Mit einer Einleitung von Wilhelm Hegeler (S. 469-476). Weimar: Klinkhardt \& Biermann.

Klemperer, V. (1996). LTI. Notizbuch eines Philologen. Leipzig: Reclam.

Kotthoff, H. (2020). Gender-Sternchen, Binnen-I oder generisches Maskulinum, ... (Akademische) Textstile der Personenreferenz als Registrierungen? Linguistic online, 103(3), 105-127. https://bop.unibe. ch/linguistik-online/issue/view/1086. Zugegriffen: 1. Okt. 2020.

Mead, G.H. (1968). Geist, Identität und Gesellschaft aus der Sicht des Sozialbehaviorismus. Mit einer Einleitung hrsg. v. C. W. Morris. Frankfurt a. M.: Suhrkamp.

Orwell, G. (1949). Nineteen eighty-four. A novel. London: Secker \& Warburg.

RdR, AG „Geschlechtergerechte Schreibung“ (2018). Bericht und Vorschläge der AG „Geschlechtergerechte Schreibung“ zur Sitzung des Rats für deutsche Rechtschreibung am 16.11.2018 - Revidierte 
Fassung aufgrund des Beschlusses des Rats vom 16.11.2018. https://www.rechtschreibrat.com/DOX/ rfdr_2018-11-28_anlage_3_bericht_ag_geschlechterger_schreibung.pdf. Zugegriffen: 1. Okt. 2020. Sapir, E. (1961). Die Sprache. Eine Einführung in das Wesen der Sprache. München: Hueber.

Sternberger, D., Storz, G., \& Süskind, W.E. (1957). Aus dem Wörterbuch des Unmenschen. Hamburg: Claassen.

Wehling, E. (2017). Framing Manual. Unser gemeinsamer, freier Rundfunk ARD. Berkeley: Berkeley International Framing Institute

Whorf, B.L. (1963). Sprache, Denken, Wirklichkeit. Beiträge zur Metalinguistik und Sprachphilosophie. Reinbek: Rowohlt.

Dr. Rudolf Stöber ist Professor für Kommunikationswissenschaft an der Otto-Friedrich-Universität Bamberg. 